\title{
The solar neighbourhood age-metallicity relation - Does it exist? ${ }^{\star, \star \star}$
}

\author{
S. Feltzing ${ }^{1}$, J. Holmberg ${ }^{1}$, and J. R. Hurley ${ }^{2}$ \\ 1 Lund Observatory, Box 43, 22100 Lund, Sweden \\ e-mail: sofia, johan@astro.lu.se \\ 2 AMNH Department of Astrophysics, 79th Street at Central Park West New York, NY, 10024-5192, USA \\ e-mail: jhurley@amnh.org
}

Received 15 August 2000 / Accepted 6 August 2001

\begin{abstract}
We derive stellar ages, from evolutionary tracks, and metallicities, from Strömgren photometry, for a sample of 5828 dwarf and sub-dwarf stars from the Hipparcos Catalogue. This stellar disk sample is used to investigate the age-metallicity diagram in the solar neighbourhood. Such diagrams are often used to derive a so called age-metallicity relation. Because of the size of our sample, we are able to quantify the impact on such diagrams, and derived relations, due to different selection effects. Some of these effects are of a more subtle sort, giving rise to erroneous conclusions. In particular we show that [1] the age-metallicity diagram is well populated at all ages and especially that old, metal-rich stars do exist, [2] the scatter in metallicity at any given age is larger than the observational errors, [3] the exclusion of cooler dwarf stars from an age-metallicity sample preferentially excludes old, metal-rich stars, depleting the upper right-hand corner of the age-metallicity diagram, [4] the distance dependence found in the Edvardsson et al. sample by Garnett \& Kobulnicky is an expected artifact due to the construction of the original sample. We conclude that, although some of it can be attributed to stellar migration in the galactic disk, a large part of the observed scatter is intrinsic to the formation processes of stars.
\end{abstract}

Key words. stars: fundamental parameters - stars: late-type - solar neighbourhood - galaxy: stellar content

\section{Introduction}

Ages and metallicities of dwarf stars in the solar neighbourhood provide a unique record of the progressive chemical enrichment of the interstellar medium where the stars formed. As time progresses the interstellar medium becomes more and more enriched in heavy elements. Thus we may expect the more recently formed stars to have a higher metallicity than those formed at an earlier epoch. By obtaining ages as well as metallicities for a representative stellar sample we should therefore be able to derive an age-metallicity relation. Such a relation would provide a strong constraint on any model of galactic chemical evolution. The picture might of course be more complicated, e.g. including infall of unprocessed gas or stellar migration. However, such processes can be modeled and the observed age-metallicity diagram would again provide a strong constraint on any model.

Are such simplistic assumptions borne out by observational evidence? The current evidence, from both new and older studies, appear to point in conflicting directions. The

Send offprint requests to: S. Feltzing,

e-mail: sofia@astro.lu.se

* Based on results from the ESA Hipparcos satellite.

$\star \star$ Full Table 1 is only available in electronic form at the CDS via anonymous $\mathrm{ftp}$ to

cdsarc.u-strasbg.fr (130.79.128.5) or via

http://cdsweb.u-strasbg.fr/cgi-bin/qcat?J/A+A/377/911 studies by Rocha-Pinto et al. (2000) and Twarog (1980a,b) do find good correlations between age and metallicity for dwarf stars in the local galactic disk. On the other hand several recent investigations appear to indicate that in fact the picture is rather more complicated with metal-rich stars being both young and old, e.g. Edvardsson et al. (1993), Carraro et al. (1998), Chen et al. (2000), and Feltzing \& Gonzalez (2001). For example in Edvardsson et al. (1993) the derived age-metallicity relation has a scatter around the mean $[\mathrm{Fe} / \mathrm{H}]$ at a given age that is four times as large as the relative error on individual $[\mathrm{Fe} / \mathrm{H}]$ values. Thus strongly indicating that the observed scatter is indeed real and not due to observational errors. As pointed out by Carraro et al. (1998) this means that the challenge for models of galactic chemical evolution has shifted from focusing on trying to reproduce an average trend, produced by binning in age and calculating mean $[\mathrm{Me} / \mathrm{H}]$ (the age-metallicity relation), to reproduce the overall trends as well as the observed scatter.

However, it should be remembered that most of the studies mentioned above include small numbers of stars, ranging from a few (Feltzing \& Gonzalez 2001) to $\sim 200$ Edvardsson et al. (1993). Several of them are concerned mainly with stellar abundance analysis which naturally limited the stellar samples in size. Furthermore, these samples were selected in order to study specific aspects of the galactic chemical evolution (Edvardsson et al. 1993; Chen et al. 2000) or a specific type of stars 
(Feltzing \& Gonzalez 2001) and are therefore not directly representative of the solar neighbourhood.

The new large database of good stellar parallaxes presented in the Hipparcos Catalogue (ESA 1997) provides a unique opportunity to investigate the age-metallicity plot using a larger number of stars. In the study presented here we investigate, using 5828 stars, the structures and trends in the age-metallicity plot.

The article is organized as follows: in Sect. 2 we describe the stellar sample used and how we determine the stellar parameters, $[\mathrm{Me} / \mathrm{H}], T_{\text {eff }}$, and age $(\tau)$. These are compared with those derived in other studies and in Sect. 2.3.3 we provide a detailed discussion of stellar ages determined from chromospheric indices. Section 3 presents and discusses selection effects in the age-metallicity plot, the implications of our findings are further discussed in Sect. 4. Finally Sect. 5 contains our conclusions.

\section{Data}

We selected all stars in the Hipparcos Catalogue (ESA 1997) that had Strömgren uvby-photometry available in the large catalogue compiled by Hauck \& Mermilliod (1998). Further restrictions on which stars would be allowed into our catalogue were imposed by the interval in $b-y, m_{1}$, and $c_{1}$ in which the calibrations of $[\mathrm{Fe} / \mathrm{H}]$ and $T_{\text {eff }}$ are valid, see Sect. 2.2. Furthermore, we required all the stars to have a relative error in the parallax less than $25 \%$. This was deemed necessary in order to be able to derive reliable ages. The need to impose this constraint will be further demonstrated in Sect. 2.3.

\subsection{Binarity}

For binary stars we cannot determine stellar ages, neither effective temperatures nor metallicities by simply using the available stellar isochrones and available calibrations of $u v b y$-photometry. To ensure that the contamination of our sample from binaries is minimized we excluded all stars that were flagged either as proved binaries or as probable binaries in the Hipparcos Catalogue (ESA 1997), e.g. all stars with CCDM number. To be as conservative as possible we excluded all stars that were either detected through the Hipparcos survey as binaries or had other indications of binarity, e.g. a stochastic solution or were marked as suspected non-single.

Of the 14112 stars for which we have $u v b y$-photometry and for which ages could be determined, 3946 stars were rejected due to being binaries or potential binaries. In this way we ensure that the stellar sample is as free as possible from binaries. Obviously undetected close binaries can still be in the sample. These can be discovered through spectroscopy.

\subsection{Metallicity and effective temperature determinations}

For each star we determine estimates of the interstellar reddening toward the star by using the model of Hakkila et al. (1997). We also apply the Lutz-Kelker correction to the magnitudes using the mean bias correction term from Koen (1992) with index $p=4$ (see Ng \& Bertelli 1998, for a discussion of the relevance of this correction to age determinations). The corrected magnitudes and colours are then used to derive metallicities and effective temperatures.

Metallicities were derived using the calibration by Schuster \& Nissen (1989). This calibration is valid for $\mathrm{G}$ dwarf stars for $0.37 \leq(b-y) \leq 0.59$ and for $\mathrm{F}$ dwarf stars for $0.22 \leq(b-y) \leq 0.38$ (their Eqs. (3) and (2) respectively).

Effective temperatures were derived using the calibration in Eq. (9) in Alonso et al. (1996). This calibration is valid for $0.25 \lesssim b-y \lesssim 0.7$.

By using these two calibrations we exclude a number of stars from further study. The red cut in $b-y$ for the Schuster \& Nissen (1989) calibration excludes a large number of late $\mathrm{K}$ dwarf stars. This, however, is not a problem for us since it is not possible to derive ages for stars that far down on the main-sequence where all ages (for a given metallicity) are degenerate. As can be seen from the limits given above it is the determination of the metallicities that imposes further cuts in our sample.

\subsubsection{Comparison with other studies - Metallicities}

We check the metallicities derived from the Strömgren photometry (hereafter denoted $[\mathrm{Me} / \mathrm{H}]$ ) by comparing them with spectroscopic measurements available in the literature (denoted $[\mathrm{Fe} / \mathrm{H}]$ ). It is especially important to make the comparison with large samples that have been homogeneously treated. We have chosen two recent large spectroscopic studies, Edvardsson et al. (1993) and Chen et al. (2000), Fig. 1.

The large catalogue by Cayrel de Strobel et al. (1997) was not considered here since this is a compilation of data from many different spectroscopic studies and although very valuable in many ways a comparison with that catalogue would not address the issue about the goodness of our metallicity determinations in a systematic way.

From the comparison we find that the systematic differences and scatters are

$[\mathrm{Me} / \mathrm{H}]_{\text {This work }}-[\mathrm{Fe} / \mathrm{H}]_{\mathrm{E} 93}=+0.01 \pm 0.10$

and

$[\mathrm{Me} / \mathrm{H}]_{\text {This work }}-[\mathrm{Fe} / \mathrm{H}]_{\text {Chen00 }}=+0.02 \pm 0.11$.

Schuster \& Nissen (1989) found that the uncertainty in their calibration of $[\mathrm{Fe} / \mathrm{H}]$ was 0.16 dex. Our results, based on a comparison of spectroscopic and photometric metallicities for a larger and more homogeneous sample 


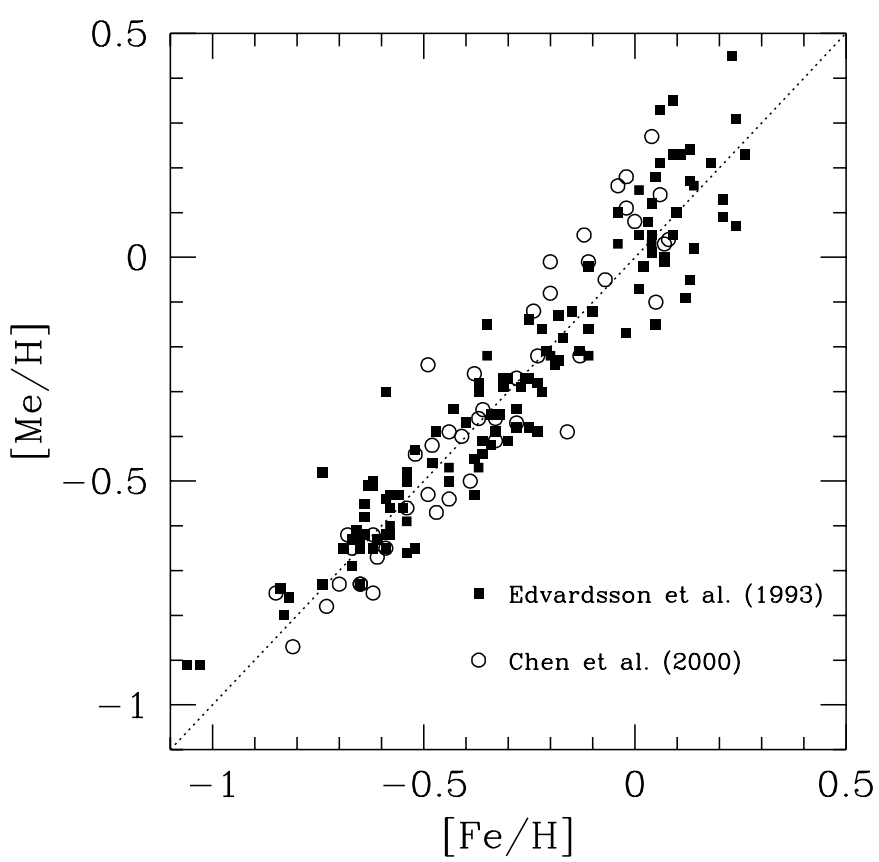

Fig. 1. Comparison of photometric $([\mathrm{Me} / \mathrm{H}])$ and spectroscopic $([\mathrm{Fe} / \mathrm{H}])$ determinations of the stellar metallicity and iron abundance respectively with Edvardsson et al. (1993) and with Chen et al. (2000).

then that used in Schuster \& Nissen (1998), in fact shows that their calibration is even better than 0.16 dex. We thus conclude that our $[\mathrm{Me} / \mathrm{H}]$ are in very good agreement with spectroscopically derived $[\mathrm{Fe} / \mathrm{H}]$ and that we can expect the errors in derived ages from errors in the $[\mathrm{Fe} / \mathrm{H}]$ determination to indeed be small.

\subsubsection{Comparison with other studies - Effective temperatures}

In Fig. 2 we compare the effective temperatures we derive using Strömgren photometry with those derived in Edvardsson et al. (1993). We find a mean difference of $-21 \mathrm{~K}$ with a scatter of $72 \mathrm{~K}$ for the 120 stars in common between our full sample and that of Edvardsson et al. (1993). Comparing with the Chen et al. (2000) sample we find a mean difference in effective temperature of $+38 \mathrm{~K}$ with a scatter of $80 \mathrm{~K}$ for the 47 stars in common. We thus feel justified in considering our effective temperatures well defined and that we can assume that our effective temperatures, as compared with spectroscopically determined values, have an error of not more than $100 \mathrm{~K}$ for the majority of the stars.

\subsection{Determination of stellar ages}

To determine the age of each star in our sample we make use of the rapid stellar evolution algorithm presented by Hurley et al. (2000). This algorithm comprises a detailed set of evolution functions which cover all evolutionary phases from the zero-age main-sequence (ZAMS) up to,

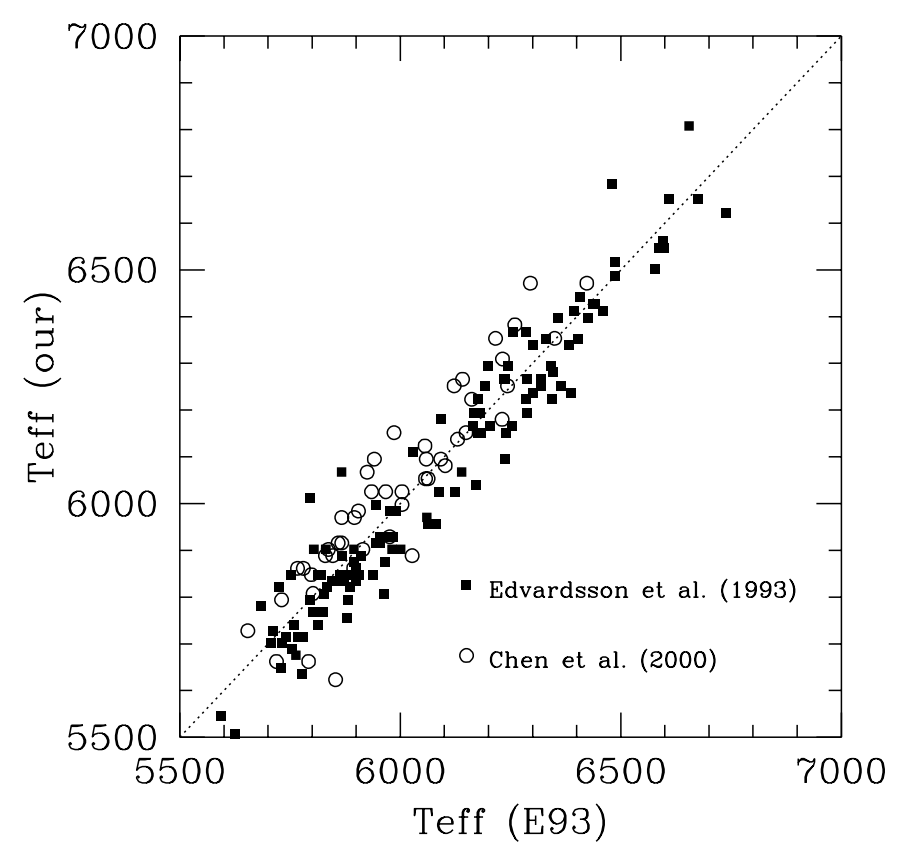

Fig. 2. Comparison of effective temperatures derived in this work and in Edvardsson et al. (1993) and with Chen et al. (2000).

and including, remnant phases such as naked helium stars and white dwarfs. All the functions are analytic, except at a few special points, and are valid for masses between 0.1 and $100 M_{\odot}$ and metallicities from $Z=10^{-4}$ to 0.03 . The functions themselves are derived from the grid of evolutionary tracks produced by Pols et al. (1998) using the detailed Eggleton evolution code (Han et al. 1994). These tracks include a modest amount of enhanced mixing beyond the classical boundary of convective instability, i.e. convective overshoot, and are approximated by the functions to within an accuracy of $5 \%$ across the entire grid. The hydrogen and helium abundances are functions of the metallicity: $X=0.76-3.0 Z$ and $Y=0.24+2.0 Z$.

For each star we have the observationally determined quantities of metal abundance, $[\mathrm{Me} / \mathrm{H}]$, absolute magnitude, $M_{V}$, and effective temperature, $\log T_{\text {eff }}$ which define a point on the Hertzsprung-Russell diagram. The stellar evolution algorithm is then used to provide a theoretical mass and age appropriate to this point by minimizing the quantity

$\epsilon=\sqrt{\left(M_{V, \mathrm{o}}-M_{V, \mathrm{t}}\right)^{2}+16\left(\log T_{\mathrm{eff}, \mathrm{o}}-\log T_{\mathrm{eff}, \mathrm{t}}\right)^{2}}$

(cf. Ng \& Bertelli 1998). The subscripts "o" and "t" refer to observational and theoretical quantities respectively. Assuming that the observational point does not lie below the ZAMS and can be reached within the maximum allowed evolution time, then for most stars $\epsilon$ is limited only by the tolerance chosen for the fitting process. This is because the evolution algorithm is continuous in mass, metallicity and age across the entire parameter space. We set the maximum allowed age to be $15.0 \mathrm{Gyr}$. We assume that all stars in our sample are either in the main-sequence 
(MS), Hertzsprung-gap or first giant branch phases of evolution, so in general the mass and age chosen is unique for the observational point. This is not always true if the point lies near the MS hook feature on an evolution track, as some overlap occurs in this region, but as this phase is rapid only a minimal error is introduced.

The appropriate bolometric corrections required for the conversion of theoretical luminosity provided by the evolution functions to $M_{V}$ are obtained from the synthetic stellar spectra computed by Kurucz (1992). We add to this an extra correction of -0.123 to account for the difference in solar offset between the models of Kurucz (1992) and Bessel et al. (1998). To convert from metal abundance to metallicity we use

$Z=0.76 /\left(3.0+37.425 \times 10^{-[\mathrm{Me} / \mathrm{H}]}\right)$

as given by Pols et al. (1998). Mass loss is included in the stellar evolution algorithm but this option is not utilized here as the rates are uncertain and most stars in our sample are not evolved enough for mass loss to noticeably affect the derived ages.

After fitting an age and a mass for each star in the standard data set we repeat the process taking the assumed errors in the data into account. These errors are $\pm 100 \mathrm{~K}$ in $T_{\text {eff }}$ and \pm 0.10 in $[\mathrm{Me} / \mathrm{H}]$. For each star we also varied the $M_{V}$ according to the $1 \sigma$ error in the parallax. This gives 7 sets of age, mass and $\epsilon$ for each star from which an average age can be calculated according to the number of good fits. We arbitrarily determine a good fit to require $\epsilon \leq 0.02$, i.e. corresponding to a fitting error of 0.005 in $\log T_{\text {eff }}$ if $M_{V, \mathrm{o}}=M_{V, \mathrm{t}}$.

\subsubsection{What is a good stellar age?}

What is a good age? Our method of determining the stellar ages allows several ways of determining this. The goodness of the fit is estimated through Eq. (1). As described in the previous section we make seven age estimates for each star. An age estimate is deemed good if $\epsilon<0.02$ which corresponds to an error in $\log T_{\text {eff }}$ of 0.005 if the input $M_{V}$ is the true $M_{V}$. The majority of the stars (8945 of 10166 stars or $88 \%$ ) have six or seven good fits. 253 of the stars have no good fit. Stars with fewer good fits are not necessarily bad. For example, a metal-rich very old star situated on the sub-giant branch might fall below the stellar evolutionary tracks when the metallicity is decreased or the magnitude increased, thus yielding a bad fit. However, such a star is still a "good" star and should be included into further considerations.

To quantify this we find the mean age for each star using only the good fits. Then we calculate the scatter around the mean age, $\sigma_{\tau}$. However, it is only the $\sigma_{\tau}$ in relation to the mean age determined that give useful information. Figure 3 shows the distribution of $\sigma_{\tau} / \tau_{\text {mean }}$ for all stars with more than 1 good fit, i.e. when the concept of $\sigma_{\tau}$ becomes meaningful. 3744 stars have $\sigma_{\tau} / \tau_{\text {mean }}>0.5$. We will use 0.5 as the dividing point between what we deem
Table 1. Stellar parameters derived in this work. $n$ indicates the number of good fits. The full table for all 5828 stars in our final sample is only available electronically at the CDS.

\begin{tabular}{rrrrrrrr}
\hline HIP & $M_{V}$ & $\log T_{\text {eff }}$ & $\begin{array}{r}{[\mathrm{Me} / \mathrm{H}]} \\
\text { dex }\end{array}$ & $\begin{array}{r}\tau_{\text {mean }} \\
\text { Gyr }\end{array}$ & $\begin{array}{r}\sigma_{\tau} \\
\text { Gyr }\end{array}$ & $n$ & $\begin{array}{r}\text { Mass } \\
M_{\odot}\end{array}$ \\
\hline 20 & 3.48 & 3.801 & -0.31 & 4.334 & 1.443 & 7 & 1.157 \\
23 & 2.90 & 3.817 & -0.36 & 2.565 & 0.478 & 7 & 1.321 \\
33 & 2.64 & 3.818 & -0.17 & 2.111 & 0.306 & 7 & 1.452 \\
39 & 2.49 & 3.817 & -0.29 & 2.180 & 0.271 & 7 & 1.455 \\
$\ldots$ & $\ldots$ & $\ldots$ & $\ldots$ & $\ldots$ & $\ldots$ & $\ldots$ & $\ldots$ \\
$\ldots$ & $\ldots$ & $\ldots$ & $\ldots$ & $\ldots$ & $\ldots$ & $\ldots$ & $\ldots$ \\
$\ldots$ & $\ldots$ & $\ldots$ & $\ldots$ & $\ldots$ & $\ldots$ & $\ldots$ & $\ldots$ \\
\hline
\end{tabular}

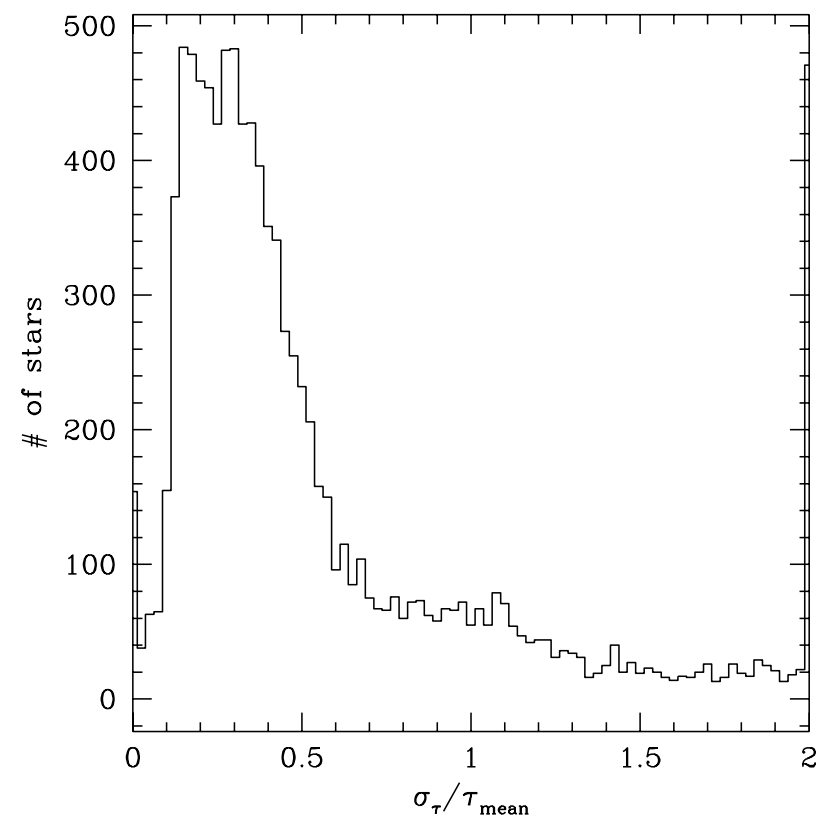

Fig. 3. Histogram of $\sigma_{\tau} / \tau_{\text {mean }}$ for all stars in the full sample, excluding the 253 stars that have no good fit. See the text for explanation of $\sigma_{\tau}$ and $\tau_{\text {mean }}$.

a good and a less good age. This number is not magical in any sense but inspection of individual stars has lead us to the conclusion that this is a reasonable cut-off. Figures 4 and 5 show the variation $\sigma_{\tau} / \tau_{\text {mean }}$ as a function of mean age and absolute magnitude. From now on we will only include stars with $\sigma_{\tau} / \tau_{\text {mean }} \leq 0.5$ and at least two good fits.

Determination of stellar ages becomes progressively more difficult and uncertain as we proceed to fainter and fainter stars in the HR-diagram because the stellar isochrones gets closer and closer. For too (absolutely) faint stars there will be a tendency to over-estimate the stellar age. To find out where our method finds its limit we have studied those Hyades stars present in our sample that are also in Perryman et al. (1998). From this we find that our ages start to become systematically older below $M_{V}>4.4$. We will henceforth take this as our lower limit for including stars into our discussions. 


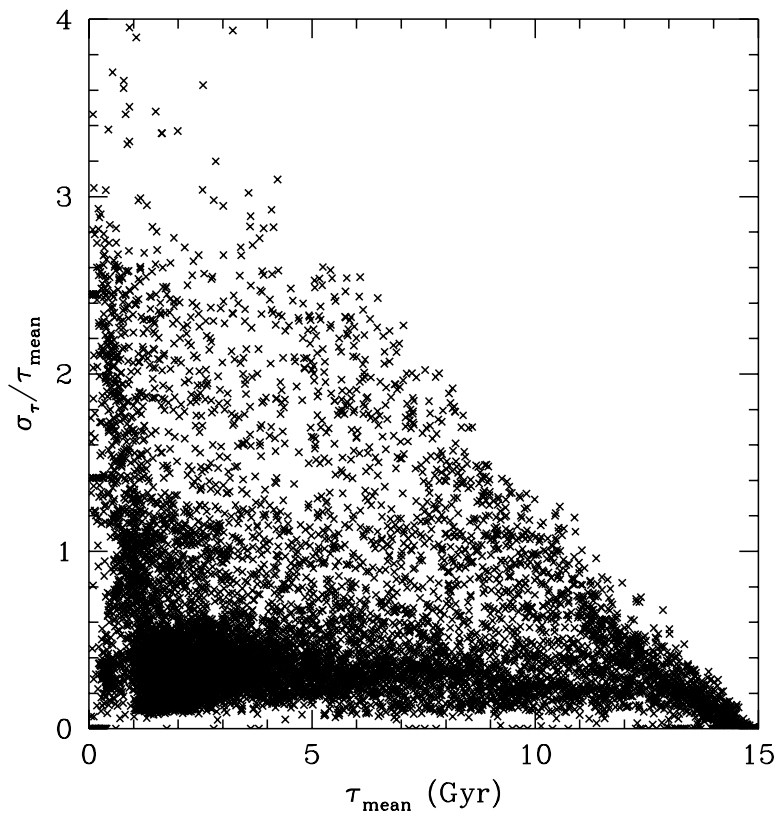

Fig. 4. $\sigma_{\tau} / \tau_{\text {mean }}$ as a function of $\tau_{\text {mean }}$ for all stars in the full sample.

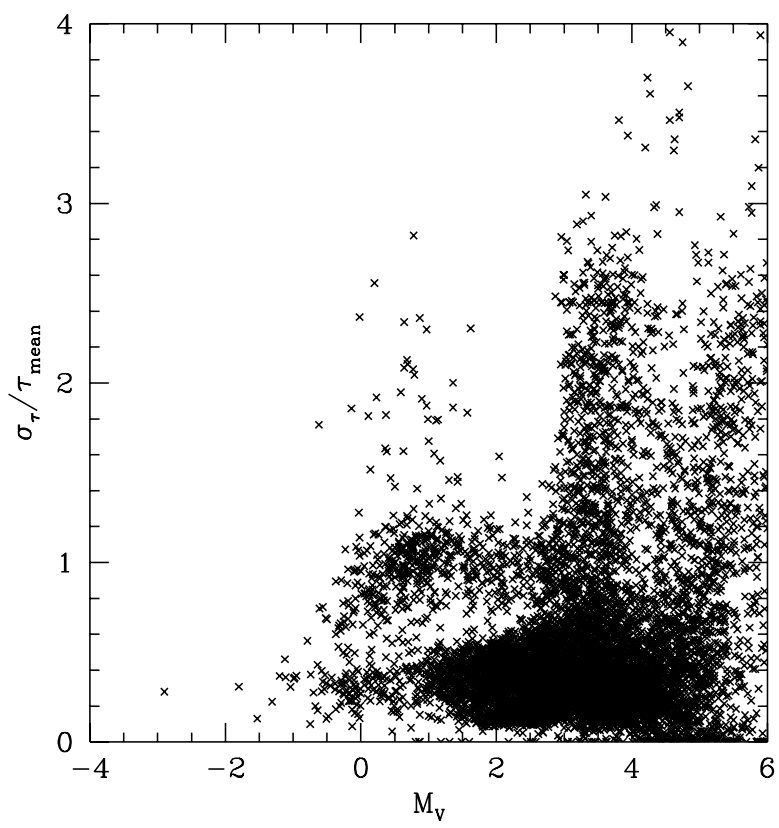

Fig. 5. $\sigma_{\tau} / \tau_{\text {mean }}$ as a function of absolute magnitude for all stars in the full sample.

\subsubsection{Comparison with $\mathrm{Ng} \&$ Bertelli (1998)}

In Fig. 6 we compare the ages derived here with those of $\mathrm{Ng} \&$ Bertelli (1998) for the Edvardsson et al. (1993) sample. This is a particularly valuable comparison since we are basically using the same method to determine our fits of the stellar tracks to the data points but use different sets of stellar evolutionary tracks, see Pols et al. (1998) and Bertelli et al. (1994). The overall agreement is good. We note that there are stars that deviate significantly from the general trend. However, the age determinations for those stars also have large errorbars and most of them would,

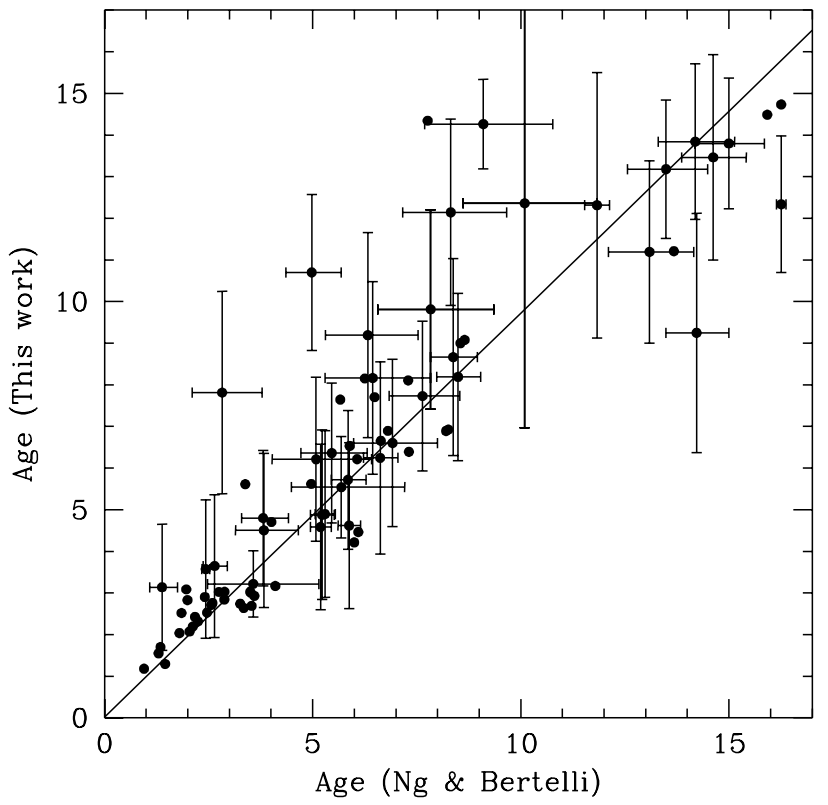

Fig. 6. Comparison of ages derived in this work and in $\mathrm{Ng} \&$ Bertelli (1998). Errors bars are indicated if they are larger than 1.5 Gyr. Only stars that have $M_{V}<4.4, \sigma_{\tau} / \tau_{\text {mean }} \leq 0.5$ and the number of good fits in both studies are larger than 1 are included. A linear least square fit, taking the indicated errors into account, is also shown.

within the estimated error, fit well into the general trend. This is especially true for the oldest stars. It is also to be expected that the oldest stars should have the largest errors on their derived ages since stellar isochrones crowd relatively more for old ages than for young.

\subsubsection{Isochrone vs. chromospheric ages}

Stellar evolutionary tracks and isochrones are not the only way to determine stellar ages. Especially the method of using chromospheric indices to date stars has recently been applied in studies of the star-formation history and agemetallicity relation of the stellar disk in the Galaxy. The results have been presented in a series of papers by RochaPinto and co-authours, e.g. Rocha-Pinto et al. (2000). In the context of the stellar ages the paper by Rocha-Pinto \& Maciel (1998) is the important paper which details the exact way the ages are determined.

In brief their method relies on one of several possible relations derived by Soderblom et al. (1991) between the $R_{\mathrm{HK}}^{\prime}$ index and the age of a star, $\tau_{\mathrm{chr}}$. This relation is, in Rocha-Pinto \& Maciel (1998), calibrated onto isochrone ages, $\tau_{\text {iso }}$. The calibration is done using 40 stars in common between their sample and that of Edvardsson et al. (1993). The more up-to-date ages from Ng \& Bertelli (1998) for the Edvardsson et al. (1993) sample were used. The difference of the $\log \tau_{\text {chr }}$ and $\log \tau_{\text {iso }}$ is found as a function of [Fe/H] (Fig. 1b in Rocha-Pinto \& Maciel 1998). This correction is then applied to the chromospheric ages for their stars. There are three points worth noting about this calibration: 1) the relation found by Soderblom et al. (1991) is by no means unique (see discussions in Soderblom et al.), 

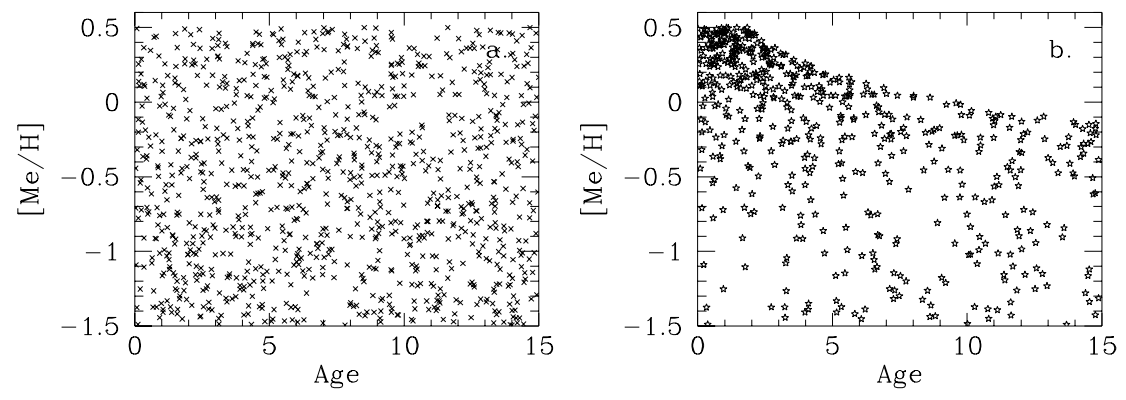

Fig. 7. These panels show an illustration of the effects on the distribution of stellar ages when the calibration from Rocha-Pinto \& Maciel (1998) is applied to a sample of stars. a) A simulated age-metallicity plot (ages derived from chromospheric indices). b) The ages corrected according to Eqs. (3) and (4) in Rocha-Pinto \& Maciel (1998). c) and d) show the respective histograms of the ages for plot $\mathbf{a})$ and $\mathbf{b})$.
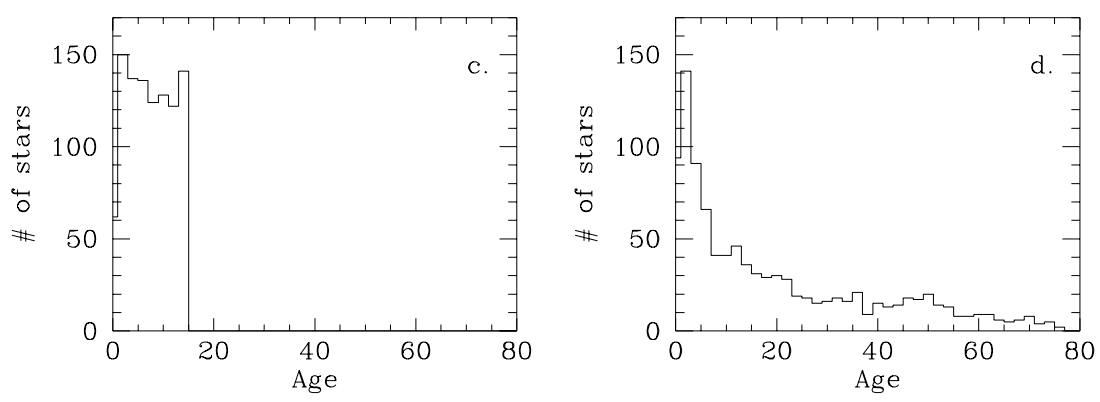

2) the calibration between the difference of $\log \tau_{\text {chr }}$ and $\log \tau_{\text {iso }}$ as a function of $[\mathrm{Fe} / \mathrm{H}]$ is not well defined, another polynomial (even a straight line) could fit equally well (no errors on the fitting are given), 3) it is not clear that the fitting formula does not suffer from selection biases, i.e. a small number of stars are involved and the scatter is large. With regards to the last point we note in particular the correction formula found in Rocha-Pinto \& Maciel (1998), because it is the $\Delta \log \tau$ that is determined, applies a correction factor to the ages such that a large age will get a large correction factor while a small age, with the same $[\mathrm{Me} / \mathrm{H}]$, will only have a small correction. Moreover, the correction formula is so constructed that no matter where the star is originally in the age-metallicity plot, old, metalrich stars will have a large correction factor, making them younger, and young, metal-poor stars will have a small correction factor, i.e. making them older. In this way all metal-rich old stars will automatically become young and the majority of young, metal-poor will become old. Thus a one-to-one age metallicity relation will automatically be found using the derived correction formula no matter how the stars are first distributed in the age-metallicity diagram.

As a simple illustration of the described effect using the corrections according to Eqs. (3) and (4) in Rocha-Pinto \& Maciel (1998) we have randomly distributed stars in an age-metallicity plot and then applied the correction factor to these (chromospheric) ages. The results are exemplified in Fig. 7. As can be seen here the correction factor moves all stars in the upper right hand corner to the left and the majority of the stars in the lower left hand corner are moved to the right. Many are moved altogether outside the plot and in Rocha-Pinto \& Maciel (1998) these stars are disregarded from further study.

We have made extensive comparisons of the ages we derive from stellar evolutionary tracks with those determined by Rocha-Pinto \& Maciel (1998). In total there are 261 stars in common between their work and that presented here. For stars with $M_{V}<4.4$ and $\sigma_{\tau} / \tau \leq 0.5$ (in our determination) we compare the chromospheric ages with our ages derived from stellar evolutionary tracks in Fig. 8.

We were especially concerned to check that our method did not give spurious old ages when in reality the star was young, as indicated by the chromospheric activity. To check this we simply plotted the stars in the HRdiagram together with stellar evolutionary tracks from Bertelli et al. (1994).

For completeness we also compare the $[\mathrm{Me} / \mathrm{H}]$ derived by us and by Rocha-Pinto \& Maciel (1998), Fig. 9. The agreement is good. The difference between the two studies is $0.06 \pm 0.09$. This is before applying corrections according to Eq. (5) in Rocha-Pinto \& Maciel (1998). However, this correction is only for chromospherically active stars (compare their Fig. 5). Most of the stars in common between their study and our are almost all inactive stars, so this correction would have little influence on the overall comparison of our results.

We want in particular to stress here that we do not say that stellar ages cannot be derived reliably from chromospheric indices. Especially for young stars this method appears very attractive and would be a much needed complement to isochrone ages since it is notoriously difficult, not to say impossible, to derive reliable isochrone ages below $\sim 1$ Gyr due to the crowding of isochrones in the HR-diagram. However, we are not equally convinced that they can be used to derive stellar ages for the oldest stars in the galactic disk and would, in fact, caution against this.

\section{The age-metallicity plot}

We now turn to consider the age-metallicity plot of local disk stars. Figure 10 shows the plot including all stars in our sample with errors in the mean less than $50 \%$ and $M_{V}<4.4$. The plot includes 5828 stars. 


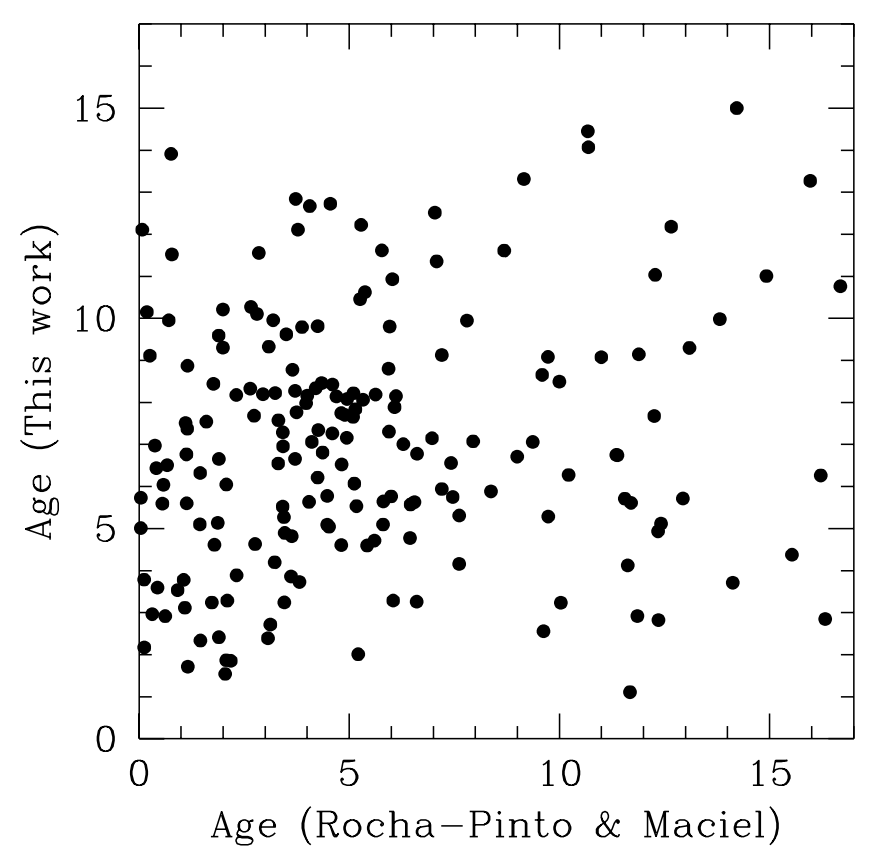

Fig. 8. Comparison of ages derived in this work and in RochaPinto \& Maciel (1998). Only stars with more than one good fit and with $M_{V}<4.4$ and $\sigma_{\tau} / \tau \leq 0.5$ are included.

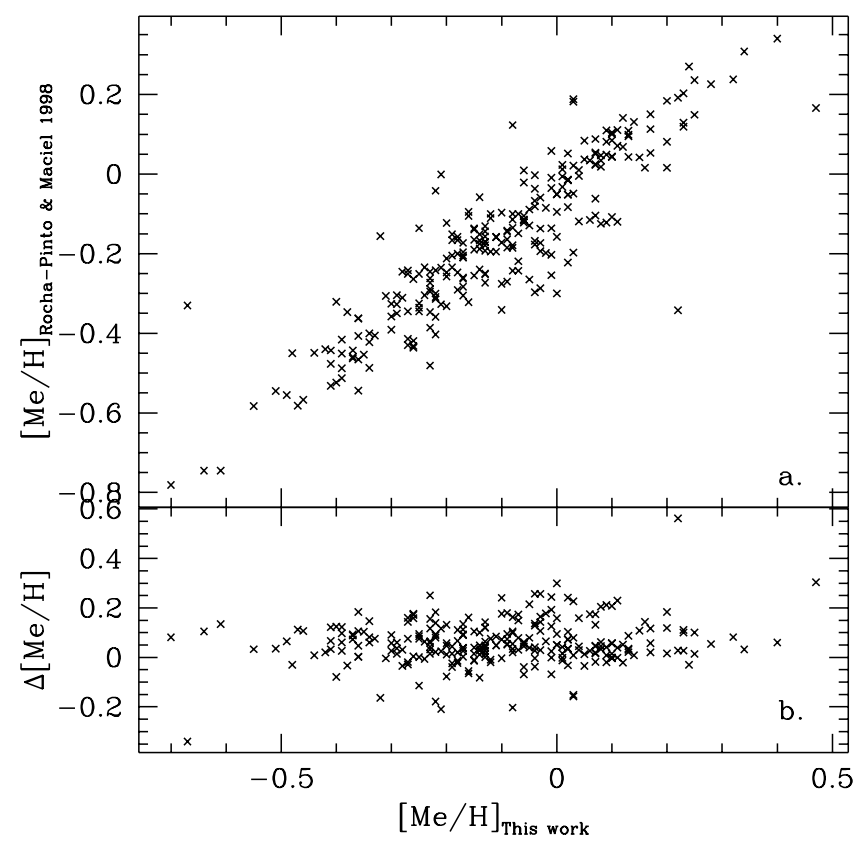

Fig. 9. Comparison of metallicities derived by us and by Rocha-Pinto \& Maciel (1998). In panel b) we show the difference between the two determinations in the sense our resultstheir results.

The main impressions from this plot are the large spread both in age and metallicity as well as a pronounced structure around 2 Gyr. Is what we are seeing the true picture or are we here subject to influences of different selection effects?

\subsection{The old, metal-rich stars}

One of the most prominent features of Fig. 10 is the very real presence of stars that are both metal-rich, i.e. $[\mathrm{Me} / \mathrm{H}]>0.0$, and old, i.e. with an age greater than 10 Gyr. The presence of such stars, if real, clearly indicates that the star formation history of the disk has been very complicated with regions (perhaps in the inner disk?) that quickly reached a high metallicity.

Some of our high age objects are at the bottom of the giant branch in the HR-diagram and would thus have somewhat more uncertain ages, even though their formal errors are small, due to the difficulties in the exact modeling of this region. However, all the stars with high metallicities and high ages are turn-off objects and are thus not susceptible to such errors in the age determination.

We have applied reddening corrections to the observed magnitudes for all our stars before deriving their stellar parameters. Our reddening estimated are based on the model by Hakkila et al. (1997). In order to check if this model has in any way systematically over-corrected the reddening for stars that are metal-rich we select all stars with $E(b-y)<0.005$, i.e. virtually negligible corrections, and plot the age-metallicity diagram for these 716 stars in Fig. 11. Although much fewer stars now appear in the plot as compared to Fig. 10 the prominent features remain. In particular we note that old and metal-rich stars remain in roughly equal numbers proving the reality of such stars.

\subsection{The $F, G$ and $K$ dwarf stars}

Figure 12 shows the HR-diagram for all the stars in Fig. 10. The major spectral classes are also indicated. Although the majority of the stars are turn-off stars we also still include a few stars that are tending towards the giant branch (i.e. $4.4<M_{V}<2$ and $\log T_{\text {eff }}<3.7$ ) as well as a number of stars at $2<M_{V}$ and $\lg T_{\text {eff }}<3.75$. We note that the latter stars all have very young ages, i.e. these are the stars that make up the small concentration of stars at $\tau_{\text {mean }} \sim 0.5$ and around $[\mathrm{Me} / \mathrm{H}]=0.05$ dex in Fig. 10 . When strong constraints on the reddening are imposed these stars are virtually all excluded. We let these stars remain in the sample for now. The stars that are close to the bottom of the giant branch all have ages larger than 10 Gyr and scatter around -0.1 dex. Some of these will remain also when stronger constraints are set on the reddening. We keep also these stars in the full sample. There are also a few stars that appear "below" the main-sequence. These are the most metal-poor stars in our sample.

Different types of selection effects are amply illustrated in Fig. 13 where we compare the age-metallicity plots of several subsamples of Fig. 10. The stars are divided into sub-groups through their effective temperature. The subgroups have been chosen as to emphasize the important differences. Figure 13a shows the stars with the highest effective temperatures, $\log T_{\text {eff }}>3.83$. These are all early F stars. Their age-metallicity plot shows a narrow feature with age and metallicity well correlated. Figure 13b shows 

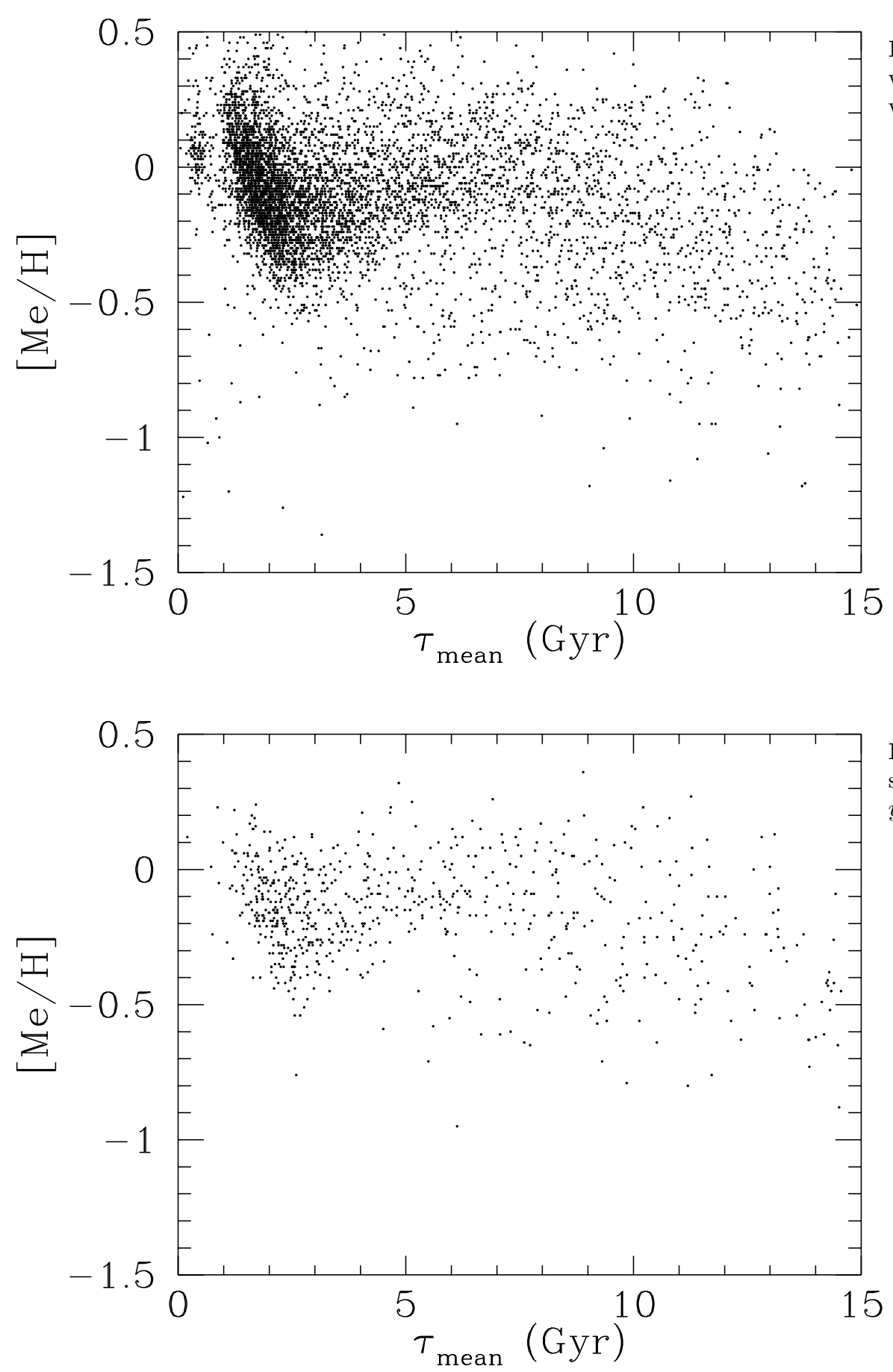

Fig. 10. The age-metallicity plot of all stars with $\sigma_{\tau} / \tau \leq 0.5$ and $M_{V}<4.4 .5828$ stars were included.
Fig. 11. The age-metallicity diagram for all stars with $\sigma_{\tau} / \tau \leq 0.5, M_{V}<4.4$, and $E(b-$ $y)<0.005 .716$ stars were included. stars in a lower temperature range, $3.83 \geq \log T_{\text {eff }}>3.8$. There is still a strong correlation between age and metallicity. If this was the stellar sample we had selected for a study of the age-metallicity relation we would derive a rather steep and strong correlation between age and metallicity. This is basically a late $\mathrm{F}$ star sample.

Figure $13 \mathrm{c}$ and $\mathrm{d}$ show perhaps the most interesting selection effects. Figure $13 \mathrm{c}$ is essentially a sample centered around spectral class G0, $3.8 \geq \log T_{\text {eff }}>3.77$, while Fig. 13d shows the same stars but also adds cooler $\mathrm{G}$ dwarf stars, $3.8 \geq \log T_{\text {eff }}>3.75$. If the first sample had been our chosen stars we would derive a fairly strong one-to-one relation between age and metallicity. If the larger sample was added, the scatter would have been increased, although the error in the mean would be lower as more stars were added, and a somewhat shallower age-metallicity relation derived.

Finally, Fig. 13e shows all stars cooler than $\log T_{\text {eff }}=$ 3.75. This includes not only dwarfs, compare the HRdiagram, but also the brightest evolved stars discussed in the first paragraph of this section. These stars all have young ages and are tracers of a stellar population, i.e. stars earlier than $\sim F 0$, that is excluded from this study due to the limitations of the calibration of $T_{\text {eff }}$. These stars 
appear in the age-metallicity plot as the small concentration of stars with around solar metallicities and have the lowest ages of all.

The most important conclusion that we reach from studying Fig. 13 is that as we allow cooler and cooler stars to enter our sample the upper right-hand corner of the age-metallicity plot is progressively filled in. This can be understood by considering the stellar isochrones in e.g. Fig. 15. As is illustrated there stellar isochrones of more metal-rich stars have a redder as well as fainter turn-off for a given age than more metal-poor stars. This means that metal-rich, old stars are excluded from the sample if we for example set the cut in $\log T_{\text {eff }}$ at $\sim 3.75$. The exact limits vary with metallicity. Also remember that the stellar sample we are discussing here only includes stars that have $M_{V}<4.4$.

Another illustration of selection effects is shown in Fig. 14. In this figure we plot the ages and metallicities for different bins of $M_{V}$. A similar pattern as in Fig. 13 appears, but now the progress from a young, confined population to a spread out is found by going to fainter and fainter samples. The selection effect is perhaps most striking in the last sub-plot where stars with $4.4<M_{V}<4$ are shown. This apparently strange selection effect is purely due to the fact that it becomes progressively harder to derive the stellar age as we move down in magnitude. Thus these stars are simply excluded through our criteria for a good stellar age. That the effect stops at younger ages for metal-rich stars than for metal-poor once again can be understood from considering the stellar isochrones in e.g. Fig. 15. From this figure we see that the isochrones in the band of absolute magnitudes between 4 and 4.4 are overall more closely packed for the metal-poor isochrones (i.e. the lower panel) than in the panel showing stellar isochrones with solar metallicities. This means that the stellar ages derived from fitting of evolutionary tracks necessarily will be more uncertain, and indeed as illustrated in Fig. 14 no stars in our sample which are in this faintest bins and have an age smaller than $\sim 10$ Gyr get an age estimate that has $\sigma_{\tau} / \tau \leq 0.5$.

From these investigations we conclude that the exact definition of the stellar sample is of the highest importance. It thus appears unlikely that a stellar sample of the solar neighbourhood can be defined such that the true age-metallicity plane is well sampled.

In studies of the age-metallicity plot and in the subsequent derivation of an age-metallicity relation the volume completeness of the sample is of prime importance. For our sample no selection according to distance was made. The important question is thus how this influences the age-metallicity plot that we have presented e.g. in Figs. 10 and 11.

For our final sample it is virtually impossible to assess the completeness due to the complex route taken to arrive at the final sample. That is we only include stars with uvby which also have a relative error in the parallax less than 0.25 . Finally we impose a cut in the relative errors of the derived ages.
Thus, instead of trying to define a subsample that is volume complete we will select stars with $E(b-y)<0.005$ and divide that sample according to the distance. This sample has a minimum of error sources for their age and metallicity determinations (see previous investigation of effect from $E(b-y))$. The distributions of distances for our full sample and that for stars with negligible reddening are shown in Fig. 16.

The age distributions we find for different distance intervals in our sample are essentially flat, Fig. 17. All distance bins show large and roughly similar scatter at all, and especially old, ages. We note that the relative number of young stars increases with distance. This is due to the fact that they are intrinsically bright and we are thus able to sample them better than the older, fainter stars. We also note that in the last distance bin the old stars have a somewhat lower mean metallicity than in the other bins. This is due to the fact that metal-poor stars, for the same age, are intrinsically brighter than the metal-rich ones and can thus be sampled more completely at larger distances.

To complete the characterization of our sample we also show the distributions of $[\mathrm{Fe} / \mathrm{H}]$ for four different age ranges in Fig. 18. The first bin contains all stars younger than 2.5 Gyr and has $\langle[\mathrm{Me} / \mathrm{H}]\rangle=-0.044$ with $\sigma$ equal to 0.19 dex. The second bin contains all stars between 2.5 and $5 \mathrm{Gyr}$ old and has $<[\mathrm{Me} / \mathrm{H}]>=-0.13$ with $\sigma$ equal to 0.20 dex. Third bin shows the stars between 5 and $10 \mathrm{Gyr}$ and has $\langle[\mathrm{Me} / \mathrm{H}]\rangle=-0.11$ with $\sigma$ equal to 0.26 dex, while the final bin contains all stars older than $10 \mathrm{Gyr}$ and has $<[\mathrm{Me} / \mathrm{H}]>=-0.31$ and a $\sigma$ of 0.33 dex. All bins only contain stars with $M_{V}<4.4$ and $\sigma_{\tau} / \tau_{\text {mean }} \leq 0.5$.

From this we may conclude that indeed the mean metallicity in the local stellar disk is high for all ages and that the scatter in metallicity at all ages also is high. It is also worth noting that the scatter in metallicity at the highest ages cannot be due to an over-estimate of the reddening correction, see Sect. 3.1 and Fig. 11, and hence we may conclude that the scatter we see is a viable representation of the underlying true metallicity distribution and that, most importantly, the scatter is at least as large as seen in diagram d of Fig. 18.

\section{Discussion - Does a unique age-metallicity relation exist?}

The previous sections presented the resulting agemetallicity plot for the solar neighbourhood using 5828 stars with well determined ages and metallicities. We also showed how different selection criteria may bias the final conclusions. We will now proceed with a discussion of previous major determinations of the age-metallicity diagram available in the literature as well as to put our results into the context of the galactic chemical evolution.

The most influential studies of the age-metallicity relation are perhaps Twarog (1980b) and Edvardsson et al. (1993). The selection of stellar samples as well as the resulting conclusions in the two papers are quite different and merits a closer look. 


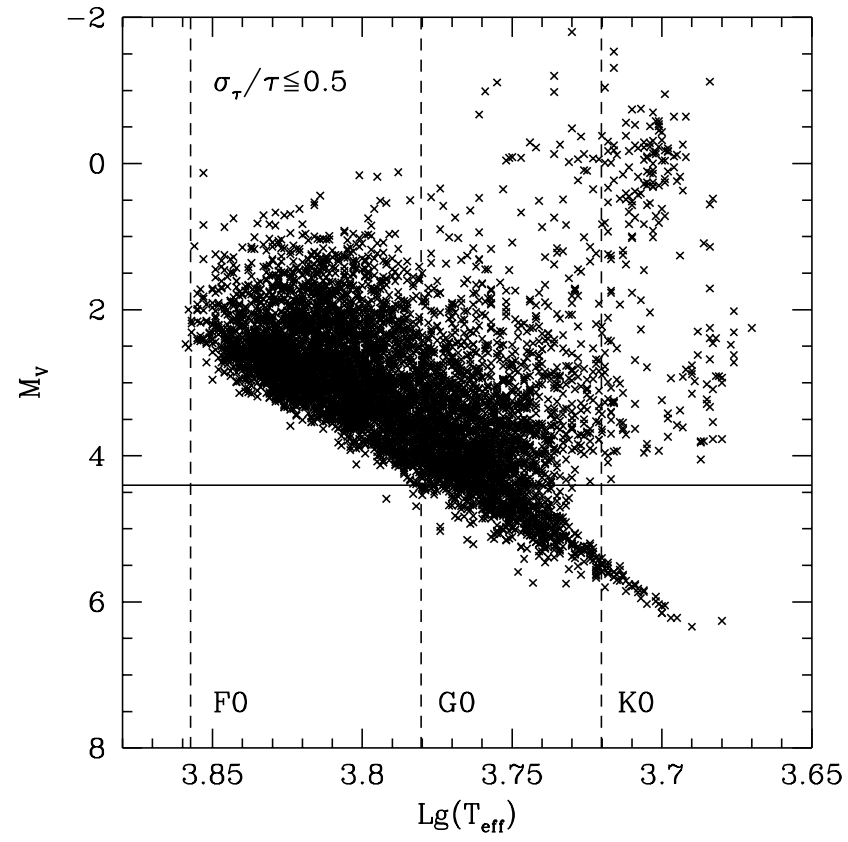

Fig. 12. The HR-diagram of all stars with $\sigma_{\tau} / \tau \leq 0.5$ and $n \geq 2$. The major spectral class divisions are also indicated (taken from Binney \& Merrifield 1998).

Twarog (1980a, 1980b) selected a stellar sample of F dwarf stars. Twarog (1980b) used a combined sample of carefully selected stars from both the Michigan catalogue as well as using the sample by Grønbech \& Olsen (1976, 1977). The stellar uvby $-\beta$ photometry was later reanalyzed in detail by Meusinger et al. (1991) using a revised calibration of stellar metallicity and new stellar isochrones from VandenBerg (1985). The total, combined sample included 536 stars (Meusinger et al. 1991). Meusinger et al. (1991) derived an age-metallicity relation similar to that of Twarog (1980b) which reflects the preconception that overall metallicity increases with time.

What are the biases in the stellar sample defined by Twarog (1980b)? First the sample is limited in apparent magnitude. Both Twarog (1980b) as well as Meusinger et al. (1991) corrected for this incompleteness when calculating the mean $[\mathrm{Me} / \mathrm{H}]$ for each age bin by weighting the $[\mathrm{Me} / \mathrm{H}]$ for each star by the inverse volume that that particular star would be visible in given its absolute magnitude. Secondly Twarog (1980b) found, through simulations, that the cuts in effective temperature should not influence the derived age-metallicity relation. Our new investigation into the effects of different cuts in effective temperature from an sample $\sim 6$ times larger than was available to Twarog shows that indeed the exact cut-off at the cooler temperature does have large effects on the appearance of the age-metallicity plot, Fig. 13. In particular old, metal-rich stars can be excluded from the sample through the lower cut in effective temperature.

In Fig. 19 we show the distributions of effective temperatures for the sample studied by Meusinger et al. (1991). Although the lower cut in effective temperature is low enough that potential old, metal-rich stars would be

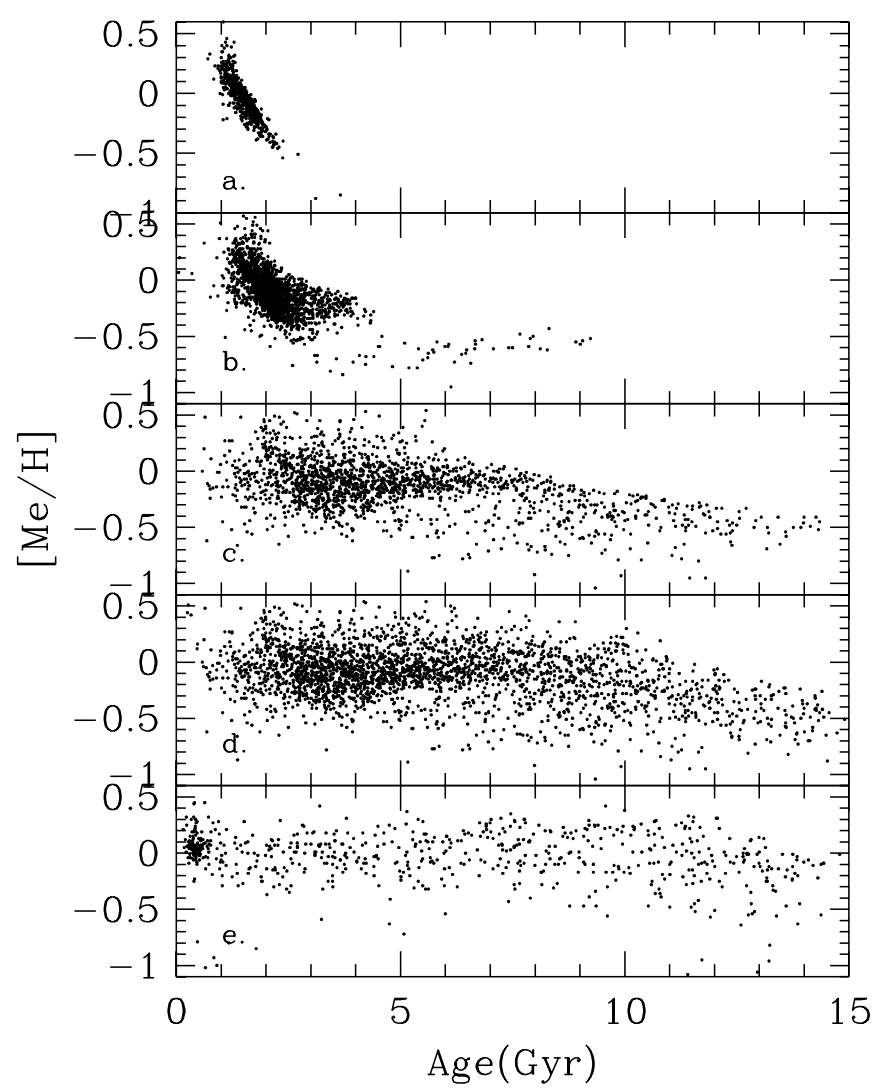

Fig. 13. Age-metallicity plots of different sub-samples from Fig. 10 and Fig. 12. a) Stars with $\log T_{\text {eff }}>3.83$, b) stars with $3.83 \geq \log T_{\text {eff }}>3.8$, c) stars with $3.8 \geq \log T_{\text {eff }}>3.77$, d) stars with $3.8 \geq \log T_{\text {eff }}>3.75$, e) stars with $3.75 \geq \log T_{\text {eff }}$. For clarity we only show $[\mathrm{Me} / \mathrm{H}]>-1$. All stars have, as before, $M_{V}<4.4$ and $\sigma_{\tau} / \tau_{\text {mean }} \leq 0.5$ and $n \geq 2$.

included the number of such stars is very small. We also compare these distributions with those of our final sample. Also, again, study the plots in Fig. 13 which shows the effect of excluding, voluntarily or un-voluntarily, colder stars. In summary the Twarog (1980b) and Meusinger et al. (1991) sample suffer from the type of selection effect that we illustrate in Fig. 13.

Although the age-metallicity relations derived by Twarog (1980b) and Meusinger et al. (1991) were successfully corrected for the magnitude bias it is apparent that if no metal-rich old stars were present in the original stellar sample then no matter what weighting schemes were invoked these stars would never be accounted for.

The Edvardsson et al. (1993) sample was, as described in detail in their article, chosen in order to study the chemical evolution of the stellar disk in the Galaxy. The stars were selected from the $u v b y-\beta$ catalogue by Olsen (1983). This catalogue contains nearly all $\mathrm{F}$ stars brighter than $V=8.3$. In nine metallicity bins, covering the metallicities of the galactic disk, 20 slightly evolved dwarf stars were chosen (for each bin) for detailed spectroscopic abundance analysis, giving an even coverage in $[\mathrm{Fe} / \mathrm{H}]$. Thus the metallicity distribution of that sample is not representative for the stellar populations of the solar neighbourhood 


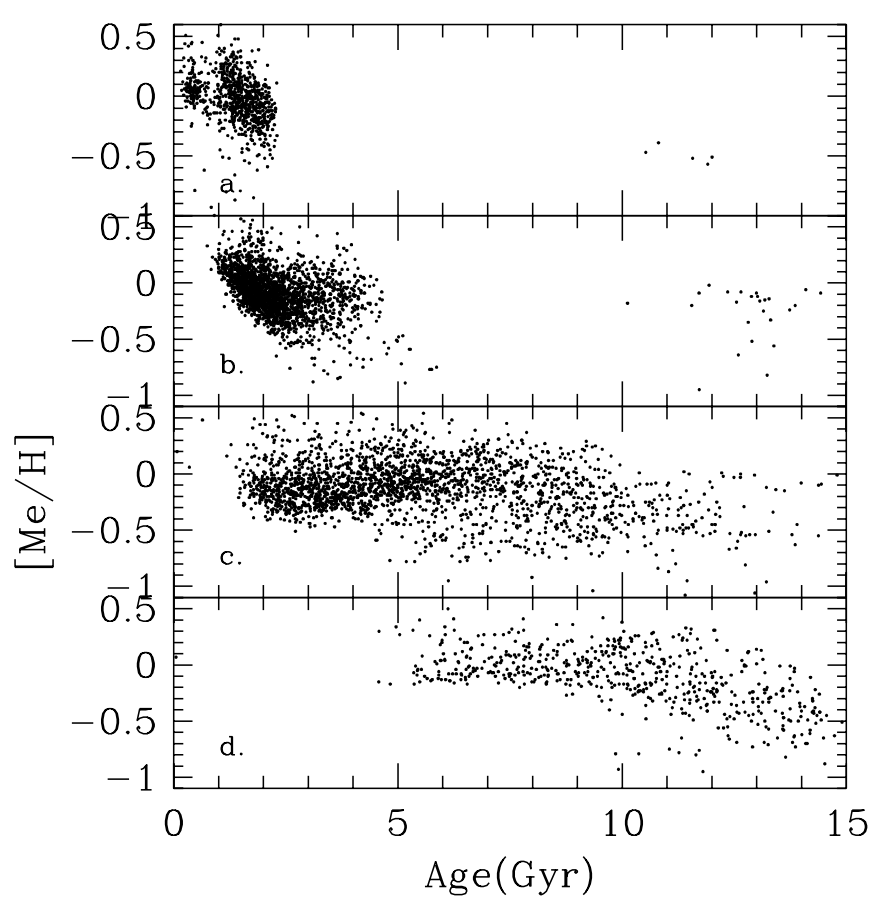

Fig. 14. Age-metallicity plots of different sub-samples from Fig. 10 and Fig. 12. a) stars with $M_{V}<2$, b) stars with $2 \leq M_{V}<3$, c) stars with $3 \leq M_{V}<4$, d) stars with $4 \leq M_{V}<4.4$. All stars have, as before, $\sigma_{\tau} / \tau_{\text {mean }} \leq 0.5$ and $n \geq 2$.

as it will necessarily include more metal-poor stars than are truly present as compared to metal-rich. They found an age-metallicity plot which showed an overall trend such that younger stars were preferentially metal-rich while old stars were preferentially metal-poor. However, once selection biases were corrected for the remaining agemetallicity relation is virtually flat between $\sim 2-10$ Gyr and has a $\sigma[\mathrm{Fe} / \mathrm{H}]=0.20$ dex. Perhaps the most important point about the Edvardsson et al. (1993) study is the high degree of internal consistency in the derived stellar abundances. In particular the small internal error on $[\mathrm{Fe} / \mathrm{H}]$ which is $\leq 0.05$ dex. This means that the scatter found in the age-metallicity plot is four times larger than the internal error on $[\mathrm{Fe} / \mathrm{H}]$ indicating that part of, or all, the scatter is real and not due to measurement errors, e.g. Nissen (1995). Furthermore, Chen et al. (2000) applied a similar abundance analysis to $90 \mathrm{~F}$ and $\mathrm{G}$ dwarf stars in the solar neighbourhood and found an equally large scatter in $[\mathrm{Fe} / \mathrm{H}]$. For example at $10 \mathrm{Gyr}$ they find a differences in $[\mathrm{Fe} / \mathrm{H}]$ of 0.8 dex between co-eval stars. In particular they conclude that there appears to exist metal-rich stars of all ages while metal-poor disk stars exclusively have old ages.

Ng \& Bertelli (1998) re-derived the stellar ages for the stars in the Edvardsson et al. (1993) sample using updated Padua isochrones (Bertelli et al. 1994) and parallaxes measured by Hipparcos (ESA 1997). We have basically used the same method as they did to derive the stellar ages (see Sect 2.3). As shown in Sect. 2.3.2 their ages and ours agree very well. $\mathrm{Ng} \&$ Bertelli (1998) found some deviation
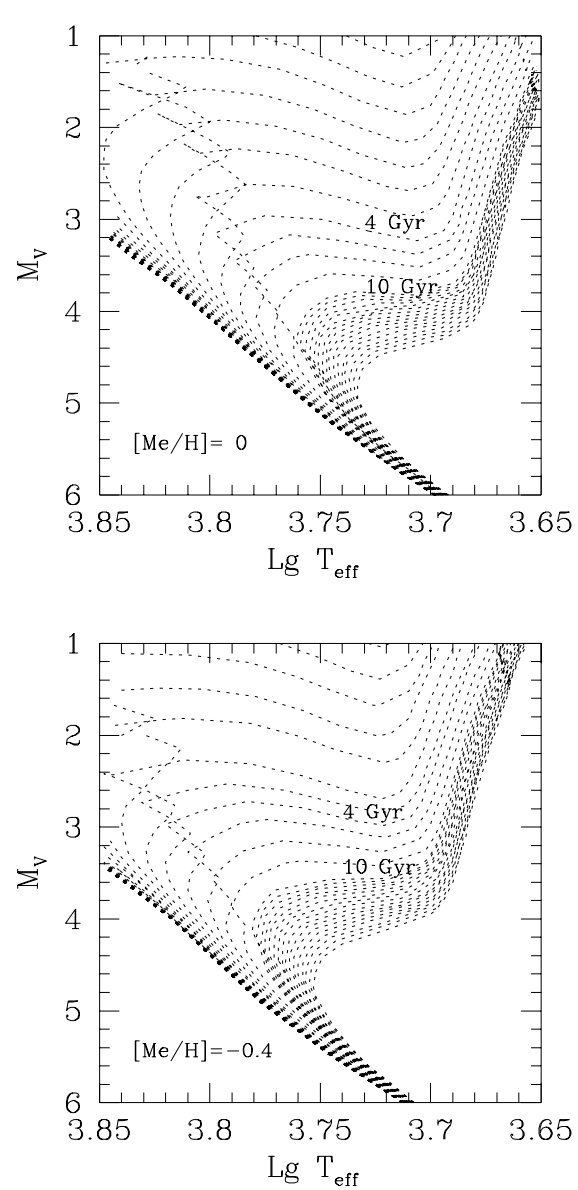

Fig. 15. Theoretical isochrones from Bertelli et al. (1994) illustrating the different turn-off colours for metallicities as indicated as well as the regions where stellar isochrones become too closely packed as to prohibit determinations of stellar ages.

in derived ages from those presented in Edvardsson et al. (1993), however, when the Hipparcos parallaxes were relied upon to determine absolute magnitudes the differences were small. Some evidence for the revised ages to be larger for old ages and somewhat younger for young ages was found but the overall impression and trends seen in the Edvardsson et al. (1993) plot were recaptured.

Garnett \& Kobulnicky (2000) divided the Edvardsson et al. (1993) sample according to distance. In their Fig. 2 they found that stars closer then $30 \mathrm{pc}$ presented a agemetallicity diagram with considerable scatter while the stars between 30 and 80 pc appeared to have tighter correlation between age and metallicity. This effect is not intrinsic to the stars in the solar neighbourhood but is expected if we consider the way the Edvardsson et al. sample was constructed. Since they were doing spectroscopic observations their sample was essentially limited in apparent magnitude. That means those stars that they selected that happened to be further away also are intrinsically more bright than those that happened to be close by. Consulting Fig. 14 we observe that when the intrinsically less bright stars are excluded then the upper-right hand corner gets depleted, i.e. metalrich, old stars are preferentially excluded. Furthermore, 


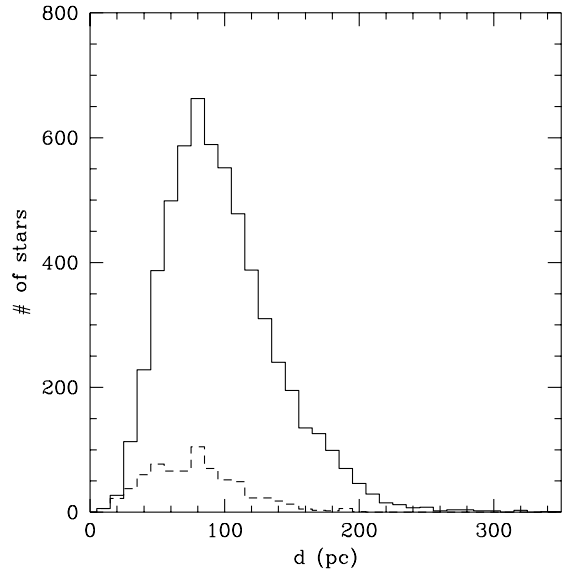

Fig. 16. Histogram of the distances for stars in our final sample (full line) and for the sub-sample of stars that have $E(b-y)<$ 0.005 .

Edvardsson et al. (1993) strived to have equal numbers of stars in each metallicity bin. As the volume density of stars with lower metallicities (i.e. $\sim-0.5$ to $-1.0 \mathrm{dex}$ ) are smaller than for the more metal-rich stars their sample naturally included many metal-poor stars that are at a greater distance from the sun. We can thus see how these two factors conspire to form a sample where metal-poor, old stars would preferentially be found in the sub-sample further away from us while the metal-rich old stars would be excluded from that sub-sample. Thus the diagrams presented by Garnett \& Kobulnicky (2000) can naturally be explained as part of the sample construction and may not be inherent to the stars in general in the solar neighbourhood.

The major conclusion from the Edvardsson et al. (1993) study and the $\mathrm{Ng}$ \& Bertelli (1998) study was that there does indeed exist a large spread in the agemetallicity plot. So large that in fact the internal measurement errors cannot account for it. In particular they found that there exist old, metal-rich stars. This is further supported by Chen et al. (2000) and Feltzing \& Gonzalez (2001), as well as by the new, larger data sample presented here.

We have already extensively compared our results with those of Rocha-Pinto et al. (2000). The distribution in $b-y$, from Fig. 1a in Rocha-Pinto et al. (2000) shows that their sample basically contains stars from $6000 \mathrm{~K}$ down to $4500 \mathrm{~K}$, although the sampling becomes progressively patchier at the lower temperatures. Thus they should not suffer from selection effects in the same way as the Twarog sample did and they should in fact have included old, metal-rich stars. Our main concern with this work and the validity of the age-metallicity relation derived stems from our investigation of the derivation of chromospheric ages, see Sect. 2.3.3.

Several models of Galactic chemical evolution have reproduced the scatter observed in Edvardsson et al. (1993) and Ng \& Bertelli (1998), e.g. Pilyugin \& Edmunds (1996), Raiteri et al. (1996), and Berczik (1999). The two latter models take into account not only the chemical

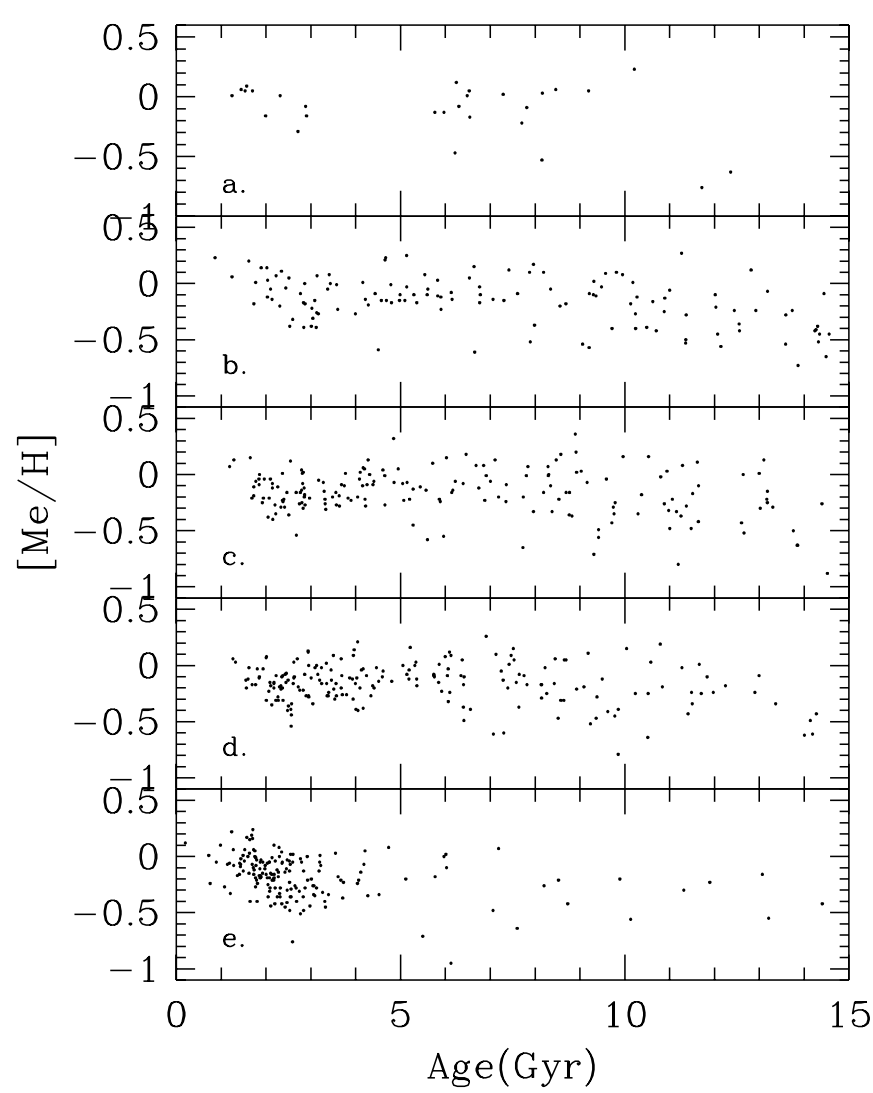

Fig. 17. Age-metallicity plots of sub-samples in different distance ranges. a) stars with $d<25$ pc, b) stars with $25 \leq$ $d<50 \mathrm{pc}$, c) stars with $50 \leq d<75$ pc, d) stars with $75 \leq d<100 \mathrm{pc}$, e) stars with $100 \leq d \mathrm{pc}$, All stars have, as before, $M_{V}<4.4$ and $\sigma_{\tau} / \tau_{\text {mean }} \leq 0.5$ and $n \geq 2$ as well as $E(b-y)<0.005$.

enrichment as time goes but also the dynamical evolution of the system, while the first model in particular studies the effect of irregular rates of infall of un-processed matter onto the stellar disk. Both approaches create a significant scatter in the age-metallicity plot supporting our conclusion that the observed scatter is real and not a result of observational errors.

\section{Conclusions}

We have presented stellar ages and metallicities for 5828 dwarf and sub-dwarf stars in the solar neighbourhood. These were used to study the age-metallicity diagram of the local stellar disk. Because of the size of the sample, to our best knowledge so far the largest sample for which such data has been presented, we were able to study, in detail, the effects of different selection criteria on the age-metallicity diagram and the conclusions drawn from such diagrams.

In particular we find that the solar neighbourhood agemetallicity diagram is well populated at all ages and especially that old, metal-rich stars do exist. Further, that exclusion of colder stars lead to a loss of old, metal-rich stars. The reason for this can be found in the stellar evolutionary tracks themselves. Old, metal-rich stars have to 


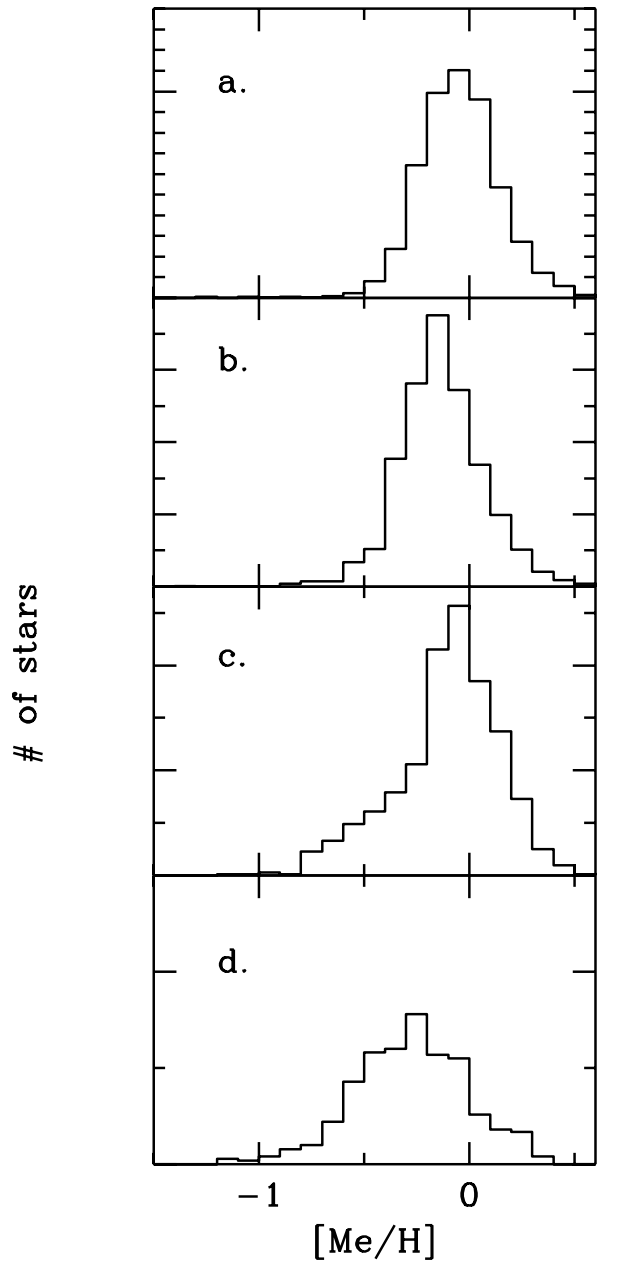

Fig. 18. Histograms for four age-ranges, a) stars with ages less than $2.5 \mathrm{Gyr}, \mathbf{b}$ ) ages between 2.5 and $5 \mathrm{Gyr}, \mathbf{c}$ ) ages between 5 and 10 Gyr, and finally d) ages larger than 10 Gyr. Note the different ranges on the $y$-axes, each tick mark, in each separate panel, corresponds to a unit of 50 stars. All stars have, as before, $M_{V}<4.4$ and $\sigma_{\tau} / \tau_{\text {mean }} \leq 0.5$ and $n \geq 2$.

be fairly evolved for stellar isochrones of different ages to be sufficiently separated in order to allow a reliable age determination. Thus it is of utmost importance when selecting a representative stellar sample to include such cold stars.

Further, we found that the scatter in metallicity at any given age are larger than the observational error, in concordance with Edvardsson et al. (1993). We also discuss how the distance dependence that Garnett \& Kobulnicky (2000) found in age-metallicity diagram of the Edvardsson et al. (1993) sample is an expected effect of the construction of their sample.

The difference between our $[\mathrm{Me} / \mathrm{H}]$ derived from uvby photometry as compared with the spectroscopic $[\mathrm{Fe} / \mathrm{H}]$ derived by Edvardsson et al. (1993) is $+0.01 \pm 0.10$ dex.

Acknowledgements. L. Lindegren is acknowledged for extensive, fruitful discussions with S. F. and J. H. The authours would like to thank the referee, W. J. Maciel, for valuable

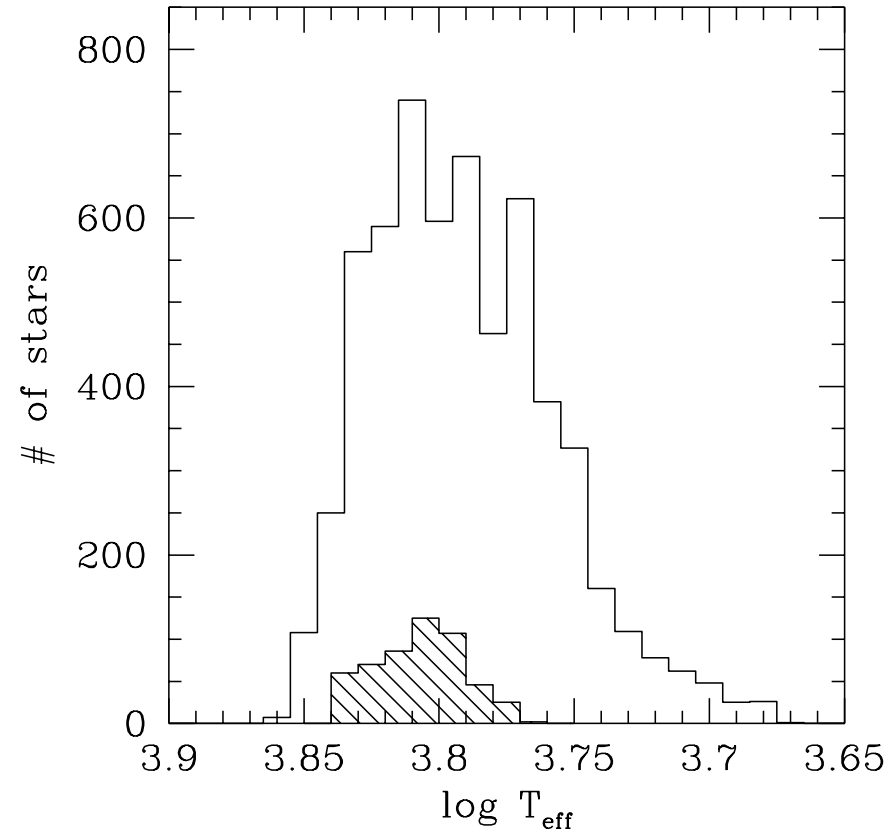

Fig. 19. Histogram showing the distribution of effective temperatures for the stars in Fig. 10. The small, shaded histogram shows the distribution for the sample in Meusinger et al. (1991) (reproduced after their Fig. 1b).

comments and suggestions that improved the article. J. Sommer-Larsen and C. Flynn are thanked for providing stimulating critisism of the text, and P. E. Nissen and B. Nordström are thanked for valuable comments and suggestions on an early draft of the paper. S. F. thanks The Royal Swedish Academy of Sciences for financial support during a stay to the Institute of Astronomy, Cambridge, UK, during which part of this work was completed. J. H. thanks the Swedish National Space Board for financial support. J. R. H. thanks G. Gilmore and the IoA for support during this work.

\section{References}

Alonso, A., Arribas, S., \& Martinez-Roger, C. 1996, A\&A, 313, 873

Berczik, P. 1999, A\&A, 348, 371

Bertelli, G., Bressan, A., Chiosi, C., Fagotto, F., \& Nasi, E. 1994, A\&AS, 106, 275

Bessell, M. S., Castelli, F., \& Plez, B. 1998, A\&A, 333, 231

Binney, J., \& Merrifield, M. 1998, in Galactic Astronomy (Princeton University Press, Princeton, New Jersey)

Carraro, G., Ng, Y. K., \& Portinari, L. 1998, MNRAS, 296, 1045

Cayrel de Strobel, G., Soubiran, C., Friel, E. D., Ralite, N., \& Francois, P. 1997, A\&AS, 124, 299

Chen, Y. Q., Nissen, P. E., Zhao, G., Zhang, H. W., \& Benoni, T. 2000, A\&AS, 141, 491

Edvardsson, B., Andersen, J., Gustafsson, B., et al. 1993, A\&A, 275,101

ESA 1997, The Hipparcos and Tycho Catalogues, ESA SP-1200

Feltzing, S., \& Gonzalez, G. 2001, A\&A, 367, 253

Garnett, D. R., \& Kobulnicky, H. A. 2000, ApJ, 532, 1192

Grønbech, B, \& Olsen, E. H. 1976, A\&AS, 25, 213

Grønbech, B, \& Olsen, E. H. 1977, A\&AS, 27, 443 
Han, Z., Podsiadlowski, P., \& Eggleton, P. P. 1994, MNRAS, 270,121

Hakkila, J., Myers, J. M., Stidham, B. J., \& Hartmann, D. H. 1997, AJ, 114, 2043

Hauck, B., \& Mermilliod, M. 1998, A\&AS, 129, 431

Hurley, J. R., Pols, O. R., \& Tout, C. A. 2000, MNRAS, 315, 543

Koen, C. 1992, MNRAS, 256, 65

Kurucz, R. L. 1992, in The Stellar Populations of Galaxies, ed. B. Barbuy, \& A. Renzini (Kluwer, Dordrecht), IAU Symp., 149,225

Meusinger, H., Stecklum, B., \& Reimann, H.-G. 1991, A\&A, 245,57

Ng, Y. K., \& Bertelli, G. 1998, A\&A, 329, 943

Nissen, P. E. 1995, in Stellar Populations, ed. P. C. van der Kruit, \& G. Gilmore (Kluwer, Dordrecht), IAU Symp., 164, 109
Olsen, E. H. 1983, A\&AS, 54, 55

Perryman, M. A. C., Brown, A. G. A., Lebreton, Y., et al. 1998, A\&A, 331, 81

Pilyugin, L. S., \& Edmunds, M. G. 1996, A\&A, 313, 792

Pols, O. R., Schröder, K-P., Hurley, J. R., Tout, C. A., \& Eggleton, P. P. 1998, MNRAS, 298, 525

Raiteri, C. M., Villata, M., \& Navarro, J. F. 1996, A\&A, 315, 105

Rocha-Pinto, H. J., \& Maciel, W. J. 1998, MNRAS, 298, 332

Rocha-Pinto, H. J., Maciel, W. J., Scalo, J., \& Flynn, C. 2000, A\&A, 358, 850

Schuster, W. J., \& Nissen, P. E. 1989, A\&A, 221, 65

Soderblom, R. D., Duncan, D. K., \& Johnson, D. R. H. 1991, ApJ, 375, 722

Twarog, B. A. 1980a, ApJS, 44, 1

Twarog, B. A. 1980b, ApJ, 242, 242

VandenBerg, D. A. 1985, ApJS, 58, 711 